\title{
Homological dimension of elementary amenable groups
}

By Peter H. Kropholler at Southampton and Conchita Martínez-Pérez at Zaragoza

\begin{abstract}
In this paper we prove that the homological dimension of an elementary amenable group over an arbitrary commutative coefficient ring is either infinite or equal to the Hirsch length of the group. Established theory gives simple group theoretical criteria for finiteness of homological dimension and so we can infer complete information about this invariant for elementary amenable groups. Stammbach proved the special case of solvable groups over coefficient fields of characteristic zero in an important paper dating from 1970.
\end{abstract}

\section{Statement of result}

We calculate the homological dimension of an elementary amenable group relative to an arbitrary coefficient ring. Throughout the paper, coefficient ring means any non-zero commutative ring. We write $\operatorname{hd}_{k}(G)$ for the homological dimension of the group $G$ over the coefficient ring $k$. When it makes sense, we write $h(G)$ for the Hirsch length of $G$. Hillman established the working definition of Hirsch length for elementary amenable groups in [11].

Theorem A. Let $G$ be an elementary amenable group and let $k$ be a coefficient ring. If $\operatorname{hd}_{k}(G)$ is finite, then $\operatorname{hd}_{k}(G)=h(G)$.

This answers a question of Bridson and the first author [6, Conjecture I.1]. Theorem A says that the homological dimension of $G$ is either equal to the Hirsch length or is infinite. Since the below proposition (which is well known) describes necessary and sufficient conditions for finiteness, it follows that we know the homological dimensions of elementary amenable groups.

Peter H. Kropholler was supported in part by Engineering and Physical Sciences Research Council grants no EP/K032208/1 and EP/N007328/1. Conchita Martínez-Pérez was supported by MINECO grant MTM2015-67781$\mathrm{P}$ and by Gobierno de Aragón and European Regional Development Funds.

Open Access (G) BY (C) 2019 Peter H. Kropholler and Conchita Martínez-Pérez, published by De Gruyter. This work is licensed under the Creative Commons Attribution 4.0 Public License. 
Proposition. Let $G$ be an elementary amenable group and let $k$ be a coefficient ring. Then $\operatorname{hd}_{k}(G)$ is finite if and only if the following two conditions hold:

(i) G has no k-torsion (meaning that the orders of elements of finite order in $G$ are invertible in $k$ ).

(ii) $h(G)<\infty$.

As observed by Fel'dman [9] one can draw the following conclusion for cohomological dimension.

Corollary A. If $G$ is a countable elementary amenable group with no $k$-torsion and with finite Hirsch length, then $h(G) \leq \operatorname{cd}_{k}(G) \leq h(G)+1$.

Proof. Over any ring, a countably generated flat module has projective dimension at most one. From this, one can deduce that the inequalities

$$
\operatorname{hd}_{k}(G) \leq \operatorname{cd}_{k}(G) \leq \operatorname{hd}_{k}(G)+1
$$

hold for any countable group $G$ with $\mathrm{hd}_{k}(G)<\infty$. Note that the inequality $h(G) \leq \operatorname{cd}_{\mathbb{Z}}(G)$ is proved in [12, Lemma 1.10 and Theorem 1.11], building on the analysis of Hillman and Linnell [13].

Subsidiary results. Along the way, we have found the need of two subsidiary results which may be of independent interest. The first is a technical splitting theorem which is essentially a refinement of a splitting theorem of Lennox-Robinson and, independently, Zaicev $[21,10.3 .2]$. Moreover, its proof uses very similar tools developed by Robinson and concerning the vanishing of certain cohomology groups for nilpotent groups (see [21, 10.3.2]).

Theorem B. Suppose that $Q$ is a group with subgroups $L, M, P$ so that the following conditions hold:

(i) $M$ and $P$ are normal in $Q$.

(ii) $L M \subseteq P$.

(iii) $M$ is a Mal'cev complete nilpotent group of finite Hirsch length.

(iv) - either $L=1$ and $P / M$ is nilpotent,

- or $L M=P$ and $L$ is nilpotent.

Then $Q$ has a subgroup $Q_{0}$ such that $L \subseteq Q_{0}, P \cap Q_{0}$ is nilpotent, and $Q_{0} M=Q$. If moreover $Q / M$ and $L$ are finitely generated, then $Q_{0}$ can be taken to be finitely generated too.

The second is a consequence of the Bieri-Strebel theory of solvable groups. For the statement we need to introduce two items of terminology. A group is called locally of type $\mathrm{FP}_{\infty}$ meaning that every finite subset of the group is contained in a subgroup of type $\mathrm{FP}_{\infty}$. As we shall see later, an elementary amenable group which is locally of type $\mathrm{FP}_{\infty}$ and which has Hirsch length $n<\infty$ always has the property that all finitely generated subgroups of Hirsch length $n$ are of type $\mathrm{FP}_{\infty}$ and this is a reason why the property has prominent role. Also, the homological dimension of solvable groups of type $\mathrm{FP}_{\infty}$ is well understood, and this is a second 
reason why the property is useful to us. Further, we say that an endomorphism $\alpha$ of an abelian group is integral if there are a positive integer $m$ and integers $b_{0}, \ldots, b_{m-1}$ such that

$$
\alpha^{m}+b_{m-1} \alpha^{m-1}+\cdots+b_{1} \alpha+b_{0}=0 .
$$

Theorem C. Let $G$ be a group with a nilpotent normal subgroup $N$ such that $h(N)<\infty$ and $G / N$ is finitely generated and abelian. Assume further that there is a finite subset $X$ of $G$ such that the following two properties hold:

(i) $G=\langle N \cup X\rangle$.

(ii) Each automorphism of $N_{\mathrm{ab}}$ that is induced by conjugation by an element of $X$ is integral.

Then $G$ is locally of type $\mathrm{FP}_{\infty}$.

Organization of the paper. In the next section we include some background material about solvable groups of finite rank, Hirsch lengths, constructible groups, and inverse duality groups. Material on nilpotent groups and their Mal'cev completions is contained in Section 3. Some reductions for Theorem A are made in Section 4. Of special note, Lemma 4.4 implies that if $G$ is an elementary amenable group with $\mathrm{hd}_{k}(G)=h(G)<\infty$, then for any subnormal subgroup $H$ of $G$ we also have $\operatorname{hd}_{k}(H)=h(H)<\infty$. Much of the technical drive in this paper concerns embedding groups as subnormal subgroups of nicer groups so that this lemma can be used.

Theorem B is proved in Section 5 using classical cohomological vanishing. Theorem C is explained in Section 6, and Theorem A is proved in a special case in Section 7. The proof of Theorem A is completed using some further applications of Theorem B in Section 8. In broad outline the idea is to embed the original group, by using Theorem B, into a group satisfying the hypotheses of Theorem $\mathrm{C}$. Then we can use established theory of solvable groups of type $\mathrm{FP}_{\infty}$ to prove Theorem A.

Acknowledgement. We are indebted to an anonymous referee for noticing a gap in our original proof of Theorem A and for showing us in detail that the argument could be corrected by using the notion of integral endomorphism. Thanks to the referee, this concept now plays a central role in the proof of the Theorem. In addition, we are grateful to him/her for many improvements to the exposition throughout the paper.

\section{Background material and historical remarks}

Recall that the class of elementary amenable groups is the smallest class of groups containing all finite and all abelian groups, that is also closed under group extensions and directed unions.

Hirsch length and homological dimension. The connection between Hirsch length and homological dimension of solvable groups was established by Stammbach who proved that hd ${ }_{K}(G)=h(G)$ whenever $G$ is solvable and $K$ is a field of characteristic zero: his elegant calculation uses exterior powers of abelian groups [22]. This work, published in 1970, was quickly followed by important work of Fel'dman, and amongst other things Fel'dman makes a claim that Stammbach's characteristic zero calculation can be extended to positive character- 
istic. However, this claim cannot be substantiated in the way that Fel'dman proposes. Bieri [3] gives a detailed account of Stammbach's result but makes no comment how a calculation of homological dimension of solvable groups in positive characteristic might proceed. In fact, modules witnessing homological dimension in positive characteristic are significantly more complicated than those used by Stammbach. Complication of some kind is unavoidable in the light of [6, Lemma I.6].

The Hillman-Linnell theorem. In the solvable case, the following result combines work of Mal'cev, Gruenberg and Robinson. We refer the reader to [21, Section 5.2] for commentary and proof. Hillman and Linnell [13] extended this to the larger class of elementary amenable groups, and for this general case we refer the reader to [12, Theorem 1.9]. Wehrfritz has given an alternative short and explicit account of this result in [23].

Lemma 2.1. Let $G$ be an elementary amenable group. If $h(G)$ is finite, then $G$ has characteristic subgroups $T \subset N \subset H \subset G$ such that the following hold:

(i) $T$ is the unique largest normal locally finite subgroup of $G$.

(ii) $N / T$ is the Fitting subgroup of $G / T$ and it is torsion-free and nilpotent.

(iii) $H / N$ is a finite rank free abelian group.

(iv) $G / H$ is finite.

The above conditions uniquely determine the subgroups $T$ and $N$. However, if $G / N$ has a non-trivial finite normal subgroup, there is not necessarily a natural condition specifying $H$ uniquely.

Another useful way of understanding Hirsch length is as follows.

Lemma 2.2. Let $G$ be an elementary amenable group. Then $G$ has finite Hirsch length if and only if there is a series $1=G_{0} \triangleleft G_{1} \triangleleft G_{2} \triangleleft \cdots \triangleleft G_{n}=G$ in which the factors are either cyclic or locally finite. Moreover, when these conditions hold, then $G$ has finitely generated subgroups with the same Hirsch length.

Proof. We comment only on the last point. Suppose that

$$
1=G_{0} \triangleleft G_{1} \triangleleft G_{2} \triangleleft \cdots \triangleleft G_{n}=G
$$

is a series with cyclic or locally finite factors. Let $J=\left\{j: G_{j} / G_{j-1} \cong \mathbb{Z}\right\}$ and for $j \in J$ choose $g_{j}$ to be a generator of $G_{j}$ modulo $G_{j-1}$. Then the subgroup $\left\langle g_{j}: j \in J\right\rangle$ is finitely generated of the same Hirsch length as $G$.

Cohomological dimension and constructible groups. Calculations of cohomological dimension for solvable groups are harder. The theory is well developed in characteristic zero and it is known that the elementary amenable groups which satisfy $\operatorname{cd}_{\mathbb{Z}}(G)=h(G)<\infty$ are precisely the torsion-free virtually solvable groups that are constructible (constructable) in the sense of Baumslag and Bieri [2]. A version of this fact was conjectured by Gildenhuys and Strebel [10] and proved by the first author [16]. Subsequently this led to a proof that elementary amenable groups of type $\mathrm{FP}_{\infty}$ over $\mathbb{Z}$ are constructible [17] and to the construction of 
classifying spaces for proper actions for such groups, see [16, 19,20]. (Results in $[17,20]$ also apply considerably beyond the elementary amenable case.)

Inverse duality groups. Key results of Fel'dman [9] are covered in Bieri's notes and are used by Brown and Geoghegan [7] to establish the following fundamental result.

Theorem 2.3 (Inverse Duality Theorem). Let G be a constructible elementary amenable group (that is, a group with a subgroup of finite index that can be built up from the trivial group with a finite number of ascending HHN-extensions). If $G$ is torsion-free, then $G$ is an inverse duality group. For such a group, it holds that $\mathrm{hd}_{k}(G)=\operatorname{cd}_{k}(G)=h(G)<\infty$ for all coefficient rings $k$.

We refer the reader to Bieri's notes for an explanation of cohomological duality and in particular the notion of inverse duality group. The inverse duality theorem holds for a wider class of groups that can be described in terms of fundamental groups of graphs of groups.

Baer's class of polyminimax groups. A virtually solvable group $G$ is called polyminimax if it has a series $1=G_{0} \triangleleft G_{1} \triangleleft \cdots \triangleleft G_{n}=G$ in which the factors are cyclic, quasicyclic or finite. Following Baer's original work [1] the term polyminimax has usually been abbreviated to minimax. Note that every virtually solvable constructible group is polymini$\max [2]$.

Locally $\mathfrak{X}$-groups. If $\mathfrak{X}$ is a class of groups or a group-theoretical property, then by a locally $\mathfrak{X}$-group we mean a group all of whose finite subsets are contained in $\mathfrak{X}$-subgroups. When $\mathfrak{X}$ is a subgroup closed class or property, then locally $\mathfrak{X}$-groups are exactly those groups whose finitely generated subgroups belong to $\mathfrak{X}$. For example, locally finite groups are groups all of whose finitely generated subgroups are finite. Locally $\mathrm{FP}_{\infty}$ groups are groups all of whose finitely generated subgroups are contained in subgroups that have type $\mathrm{FP}_{\infty}$. But note that a locally $\mathrm{FP}_{\infty}$ group can have finitely generated subgroups that are not of type $\mathrm{FP}_{\infty}$.

Commensurate subgroups. Two subgroups $H$ and $K$ of a group $G$ are said to be commensurate if $H \cap K$ has finite index in both $H$ and $K$.

Lemma 2.4. Let $G$ be an elementary amenable group of Hirsch length $n<\infty$ that is locally polyminimax. Then $G$ has finitely generated subgroups of the Hirsch length $n$ and all such subgroups are commensurate with each other.

Proof. Suppose first that $G$ is a finitely generated polyminimax group and that $H$ is a subgroup of Hirsch length $n$. A result of Robinson which uses the fact that $G$ is virtually solvable with finite abelian ranks (see [8, Theorem 3.1]) shows that $H$ has finite index in $G$ in this case.

In general, using Lemma 2.2 we can choose a finitely generated subgroup $H$ of $G$ with $h(H)=h(G)$. If $F$ is any finite subset of $G$, then

$$
h(G)=h(H) \leq h(\langle H \cup F\rangle) \leq h(G)
$$

so $h(H)=h(\langle H \cup F\rangle)$ and applying the above argument to the subgroup $H$ of the finitely 
generated group $\langle H \cup F\rangle$ we deduce that $H$ has finite index in $\langle H \cup F\rangle$. Now if $K$ is any other finitely generated subgroup of $G$ of the same Hirsch length, then we can apply this argument to $\langle H \cup K\rangle$ to deduce that $H$ and also $K$ both have finite index in $\langle H \cup K\rangle$. In particular, it follows that $H$ and $K$ are commensurate.

Putting Lemma 2.4 together with the elementary observation that the $\mathrm{FP}_{\infty}$ property of a subgroup is inherited by any commensurate subgroup, we deduce the following.

Corollary 2.5. Let $G$ be an elementary amenable group of Hirsch length $n<\infty$ that is locally of type $\mathrm{FP}_{\infty}$. Then all the finitely generated subgroups of Hirsch length $n$ are of type $\mathrm{FP}_{\infty}$ and are commensurate with each other.

\section{Nilpotent groups}

We write $\gamma_{i}(G)$ for the $i$ th term of the lower central series of $G$. It is defined inductively by $\gamma_{1}(G)=G$ and then by taking commutators:

$$
\gamma_{i+1}(G)=\left[\gamma_{i}(G), G\right] .
$$

We use a right-handed convention for commutators, namely for group elements $x$ and $y$, we write

$$
[x, y]:=x^{-1} y^{-1} x y .
$$

The free nilpotent group on $d$ generators of class $c$ is defined to be $F / \gamma_{c+1}(F)$, where $F$ is the free group on $d$ generators. So long as $d \geq 2$, this group does indeed have class $c$. Of course if $d \leq 1$, then it is cyclic. Note that there is no particular reason for $d$ to be finite: the theory makes sense for any cardinal number $d$. The class $c$ is always a non-negative integer.

Every nilpotent group $G$ has a Mal'cev completion denoted by $G^{\mathbb{Q}}$ which is uniquely determined, [21, 2.1.1]. The theory is further developed in [21, Section 2.1]. There is a natural map from $G$ to its Mal'cev completion and this is injective precisely when $G$ is torsion-free. The free nilpotent groups are torsion-free and so embed into their Mal'cev completions. The Mal'cev completion is functorial in the sense that a homomorphism $G \rightarrow H$ from one nilpotent group to another induces a uniquely determined homomorphism $G^{\mathbb{Q}} \rightarrow H^{\mathbb{Q}}$ between their Mal'cev completions. For further information we refer the reader to Hilton's paper [14]. In particular, Hilton provides a proof that there are natural isomorphisms

$$
\left(\gamma_{i}(N) / \gamma_{i+1}(N)\right)^{\mathbb{Q}} \cong \gamma_{i}\left(N^{\mathbb{Q}}\right) / \gamma_{i+1}\left(N^{\mathbb{Q}}\right)
$$

for $N$ nilpotent and $i \geq 1$.

Definition 3.1. By a free Mal'cev complete group on $d$ generators of class $c$ we shall mean the Mal'cev completion of the free nilpotent group on $d$ generators of class $c$. Whenever we use the term Mal'cev complete it is to be understood that the group in question is nilpotent.

The following is well known.

Lemma 3.2. Let $G$ be a free Mal'cev complete group of class $c$. Let $H \rightarrow K$ be any surjective homomorphism, where $H$ is Mal'cev complete of class at most c. Then every homomorphism from $G$ to $K$ factors through $H$. 
Proof. Let $d$ be the dimension of the rational vector space $G / \gamma_{2}(G)$. Let $F$ be a free nilpotent group of class $c$ on $d$ generators. We can identify $G$ with $F^{\mathbb{Q}}$. The universal property of $F$ ensures that the composite map $F \rightarrow F^{\mathbb{Q}}=G \rightarrow K$ factors through $H$. On passing to Mal'cev completion we have an induced map $F^{\mathbb{Q}}=G \rightarrow H^{\mathbb{Q}}=H$ as required.

The following lemma, also well known, is required for the proof of an important result about Mal'cev complete nilpotent groups, namely Proposition 3.6 below.

Lemma 3.3. Let $G$ be a nilpotent group and $H$ a subgroup such that $H \gamma_{2}(G)=G$. Then $H=G$.

Proof. Since $G$ is nilpotent, $H$ is subnormal and so we may replace $H$ by its normal closure and without loss of generality we may assume that $H$ is normal. The quotient $G / H$ is therefore both perfect and nilpotent which implies $H=G$.

Definition 3.4. For any group $G$ and any positive integer $m$ we say that an automorphism $\phi$ of $G$ is $m$-powering provided that for all $g \in G$,

$$
g^{\phi} \in g^{m} \gamma_{2}(G) \text {. }
$$

By a powering automorphism we mean an automorphism that is $m$-powering for some $m \geq 1$. When $G$ is a group such that $G / \gamma_{2}(G)$ is not a torsion group, then a powering automorphism is $m$-powering for a uniquely determined rational number $m$. Note that this uniqueness property therefore holds when $G$ is a non-trivial Mal'cev complete group.

Let $N$ be a group and denote by $U$ the group of 1-powering automorphisms of $N$. For each $j$ and each automorphism $\phi$ of $N$, let ${ }^{j} \phi$ denote the induced automorphism of $N / \gamma_{j+1}(N)$. The assignment $\phi \mapsto{ }^{j} \phi$ determines a homomorphism

$$
U \rightarrow \operatorname{Aut}\left(N / \gamma_{j+1}(N)\right)
$$

and we write $U_{j}$ for the kernel of this homomorphism. In this way we obtain a descending chain

$$
U=U_{1} \geq U_{2} \geq U_{3} \geq \cdots
$$

of normal subgroups of $U$. With this notation we have:

Proposition 3.5. Let $N$ be a group and let $\Gamma=\operatorname{Aut}(N)$. Let $U$ be the group of 1-powering automorphisms of $N$. For each $j \geq 1$ let $U_{j}$ denote the subgroup of $\Gamma$ comprising all automorphisms that induce the identity automorphism of $N / \gamma_{j+1}(N)$. The series

$$
U=U_{1} \geq U_{2} \geq \cdots
$$

is a descending central series of normal subgroups of $\Gamma$. Moreover, using the left $\Gamma$-module structure on $U_{j} / U_{j+1}$ arising from conjugation, $U_{j} / U_{j+1}$ is isomorphic to a submodule of

$$
\operatorname{Hom}\left(N_{\mathrm{ab}}, \gamma_{j+1}(N) / \gamma_{j+2}(N)\right)
$$

regarded as a left $\Gamma$-module using the diagonal action. It follows that if $N$ is nilpotent of class $c \geq 1$, then $U$ is nilpotent of class $c-1$. 
Proof. Let $\alpha \in U_{j}$ and denote by $\alpha^{*}$ the automorphism of $N / \gamma_{j+2}(N)$ induced by $\alpha$. The epimorphism from the $(j+1)$ st tensor power of $N_{\mathrm{ab}}$ to $\gamma_{j+1}(N) / \gamma_{j+2}(N)$ induced by the iterated commutator map reveals that $\alpha$ induces the identity map on $\gamma_{j+1}(N) / \gamma_{j+2}(N)$. That is to say that the diagram

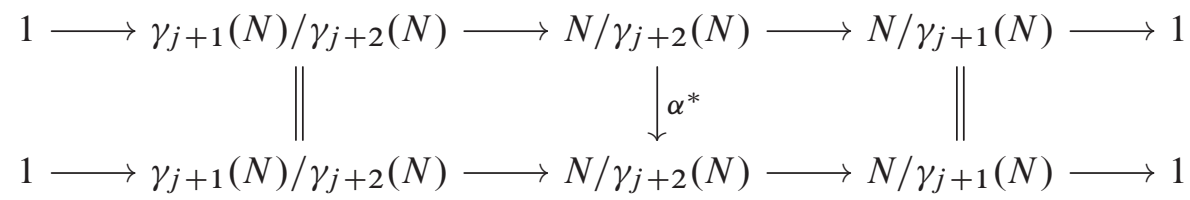

commutes. Now define the homomorphism $\phi_{\alpha}: N_{\mathrm{ab}} \rightarrow \gamma_{j+1}(N) / \gamma_{j+2}(N)$ by

$$
\phi_{\alpha}(g[N, N])=\alpha(g) g^{-1} \gamma_{j+2}(N) .
$$

It can then be verified that the map $\alpha \mapsto \phi_{\alpha}$ defines a homomorphism

$$
U_{j} \rightarrow \operatorname{Hom}\left(N_{\mathrm{ab}}, \gamma_{j+1}(N) / \gamma_{j+2}(N)\right)
$$

of $\Gamma$-operator groups whose kernel is $U_{j+1}$. Moreover, since the group $U$ acts trivially on $\operatorname{Hom}\left(N_{\mathrm{ab}}, \gamma_{j+1}(N) / \gamma_{j+2}(N)\right)$, the series $U=U_{1} \geq U_{2} \geq \cdots$ is central. Hence, if $N$ is nilpotent of class $c \geq 1$, then $U$ is also nilpotent and has class $\leq c-1$. Since $U$ contains a copy of $N / Z(N)$, we conclude that $U$ has class exactly $c-1$.

For a group $G$ and a subgroup $H$, let $\operatorname{Aut}(G ; H)$ denote the set of those automorphisms of $G$ which restrict to automorphisms of $H$. This is a subgroup of $\operatorname{Aut}(G)$ and the restriction map affords a homomorphism $\operatorname{Aut}(G ; H) \rightarrow \operatorname{Aut}(H)$. If $H$ is normal in $G$, then elements of $\operatorname{Aut}(G ; H)$ naturally induce automorphisms of $G / H$ and there is a homomorphism $\operatorname{Aut}(G ; H) \rightarrow \operatorname{Aut}(G / H)$.

Proposition 3.6. Let $N$ be a Mal'cev complete nilpotent group with $h(N)<\infty$. Then there exist a free Mal'cev complete nilpotent group $\widehat{N}$ of the same class as $N$ and an epimorphism $\pi: \widehat{N} \rightarrow N$ such that the following two statements hold:

(i) The induced map $\pi_{\mathrm{ab}}: \widehat{N}_{\mathrm{ab}} \rightarrow N_{\mathrm{ab}}$ is an isomorphism.

(ii) The induced homomorphism $\bar{\pi}: \operatorname{Aut}(\widehat{N} ; \operatorname{Ker} \pi) \rightarrow \operatorname{Aut}(N)$ is surjective, and the inverse image under $\bar{\pi}$ of the subgroup of 1-powering automorphisms of $N$ is the subgroup of all 1-powering automorphisms in $\operatorname{Aut}(\widehat{N} ; \operatorname{Ker} \pi)$.

Proof. Let $d=\operatorname{dim}_{\mathbb{Q}} N_{\mathrm{ab}}$ and let $c$ be the class of $N$. Take $F$ to be the free nilpotent group of class $c$ on a generating set $\delta$ of cardinality $d$. Also, let $\mathcal{T}$ be a subset of $N$ with cardinality $d$ whose image in $N_{\text {ab }}$ is a $\mathbb{Q}$-basis of $N_{\text {ab. Set }} \widehat{N}:=F^{\mathbb{Q}}$. Fix a bijection from $\delta$ to $\mathcal{T}$ : this gives rise to a unique homomorphism $F \rightarrow N$. The universal property of Mal'cev completions now yields a homomorphism $\pi: \widehat{N} \rightarrow N$ satisfying property (i) and this is surjective by Lemma 3.3.

For an automorphism $\alpha \in \operatorname{Aut}(N)$, the projective property (Lemma 3.2) supplies a homomorphism $\widehat{\alpha}: \widehat{N} \rightarrow \widehat{N}$ so that the diagram

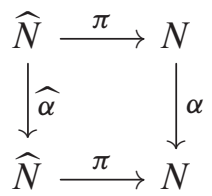


commutes. By considering the abelianization of this commutative diagram, we see that $\widehat{\alpha}_{\mathrm{ab}}$ is an automorphism. Thus, by Lemma 3.3, $\widehat{\alpha}$ is surjective and so also injective. This implies that $\widehat{\alpha} \in \operatorname{Aut}(\widehat{N} ; \operatorname{Ker} \pi)$ and $\bar{\pi}(\widehat{\alpha})=\alpha$. Thus $\bar{\pi}$ is surjective. In addition, the abelianization of the diagram reveals that $\widehat{\alpha}_{\mathrm{ab}}$ is the identity map on $\widehat{N}_{\mathrm{ab}}$ if and only if $\alpha_{\mathrm{ab}}$ is the identity map on $N_{\mathrm{ab}}$. Statement (ii) is now proved.

The following result will be useful for our arguments.

Lemma 3.7. Let $N$ be a nilpotent group and $U$ the group of 1-powering automorphisms of $N$. Let $\Gamma$ be a subgroup of $\operatorname{Aut}(N)$ that acts nilpotently on $N$.

(i) If $N$ is torsion-free, then so is $\Gamma$.

(ii) If $N$ is Mal'cev complete, then so is $U$.

Proof. We give a brief outline. For (i) we can use the upper central series of $N$ to reduce to the case when $N$ is torsion-free and abelian in which case the automorphisms that act nilpotently behave like upper unitriangular matrices and clearly have infinite order whenever non-trivial. For (ii) one can also use induction on the length of the upper central series of $N$.

The next lemma illustrates that Mal'cev complete groups have an abundance of powering automorphisms, and is an easy consequence of Lemma 3.2. It plays a vital role in this paper.

Lemma 3.8. Let $m$ be a positive integer and let $F$ be a free Mal'cev complete group. Then there exists an m-powering automorphism of $F$.

\section{Reductions for the proof of Theorem A}

Locally $\mathrm{FP}_{\infty}$ groups have an important role to play in our arguments. Before returning to this class we need to mention the key inequalities of a more elementary nature.

Lemma 4.1 ([6, Theorem I.2]). Let $G$ be an elementary amenable group. Then the following are equivalent:

(i) $\operatorname{hd}_{k}(G)<\infty$.

(ii) $h(G)<\infty$ and $G$ has no $k$-torsion.

When these conditions hold, we have $\frac{h(G)}{2} \leq \operatorname{hd}_{k}(G) \leq h(G)$.

We refer the reader to [3] for the standard theory summarized in the following proposition.

Proposition 4.2. The following hold for any group $G$ and any coefficient ring $k$ such that $\operatorname{hd}_{k}(G)<\infty$.

(i) If $H$ is a subgroup of $G$, then $\operatorname{hd}_{k}(G) \geq \operatorname{hd}_{k}(H)$.

(ii) If $H$ has finite index in $G$, then $\operatorname{hd}_{k}(G)=\operatorname{hd}_{k}(H)$. 
(iii) If $T$ is a normal locally finite subgroup of $G$, then $\operatorname{hd}_{k}(G / T)=\operatorname{hd}_{k}(G)$.

(iv) If $H$ is a subgroup with the property that $|\langle H \cup F\rangle: H|<\infty$ for all finite subsets of $G$, then $\operatorname{hd}_{k}(G)=\operatorname{hd}_{k}(H)$.

We now return to groups that are locally of type $\mathrm{FP}_{\infty}$. The following result is fundamental to our approach to Theorem A.

Proposition 4.3. Let $G$ be an elementary amenable group that is locally of type $\mathrm{FP}_{\infty}$. If $\operatorname{hd}_{k}(G)<\infty$, then $\operatorname{hd}_{k}(G)=h(G)$.

Proof. Assume that $\mathrm{hd}_{k}(G)$ is finite. Then $G$ has no $k$-torsion and $h(G)$ is finite. Let $H$ be a finitely generated subgroup of $G$ of the same Hirsch length. Then $H$ is of type $\mathrm{FP}_{\infty}$ by Corollary 2.5. Therefore $H$ is constructible and is virtually an inverse duality group over $\mathbb{Z}$. It follows that $h(G)=h(H)=\operatorname{hd}_{k}(H) \leq \operatorname{hd}_{k}(G)$. Lemma 4.1 gives the reverse inequality $\operatorname{hd}_{k}(G) \leq h(G)$ and we deduce that $\operatorname{hd}_{k}(G)=h(G)$.

Finally in this section, we record a simple spectral sequence argument which can be used to show that the validity of the conclusion of Theorem A is inherited by subnormal subgroups.

Lemma 4.4. Let $G$ be an elementary amenable group with $\mathrm{hd}_{k}(G)=h(G)<\infty$ and let $N$ be a normal subgroup of $G$ such that $\operatorname{hd}_{k}(G / N)<\infty$. Then $\operatorname{hd}_{k}(G / N)=h(G / N)$ and $\operatorname{hd}_{k}(N)=h(N)$. Furthermore, if $N$ is any normal subgroup of $G$, then the conclusion $\operatorname{hd}_{k}(N)=h(N)$ holds whether or not $\operatorname{hd}_{k}(G / N)<\infty$.

Proof. By the Lyndon-Hochshild-Serre spectral sequence we have

$$
\operatorname{hd}_{k}(G) \leq \operatorname{hd}_{k}(G / N)+\operatorname{hd}_{k}(N) .
$$

By Proposition 4.2 (i) we know $\operatorname{hd}_{k}(N)<\infty$ and so Lemma 4.1 gives

$$
\operatorname{hd}_{k}(G / N) \leq h(G / N) \text { and } \operatorname{hd}_{k}(N) \leq h(N) .
$$

Putting the pieces together, we have

$$
h(G)=\operatorname{hd}_{k}(G) \leq \operatorname{hd}_{k}(G / N)+\operatorname{hd}_{k}(N) \leq h(G / N)+h(N)=h(G)
$$

which gives the desired result in case $\operatorname{hd}_{k}(G / N)<\infty$. In general, $G / N$ may not have finite homological dimension, but it is an elementary amenable group of finite Hirsch length and by Lemma 2.1 there exist subgroups $N_{1} \triangleleft G_{1}$ such that $N \leq N_{1}, G_{1}$ has finite index in $G, N_{1} / N$ is locally finite, and $G_{1} / N_{1}$ is torsion-free. By Proposition 4.2 (iv), $\operatorname{hd}_{k}(N)=\operatorname{hd}_{k}\left(N_{1}\right)$ and by Proposition 4.2 (ii), $\operatorname{hd}_{k}(G)=\operatorname{hd}_{k}\left(G_{1}\right)$. Note also that $N$ and $N_{1}$ have the same Hirsch length.

Now we can apply the above argument to the situation that $N_{1}$ is a normal subgroup of $G_{1}$ because $G_{1} / N_{1}$ being torsion-free of finite Hirsch length does indeed have finite homological dimension. This shows that $\mathrm{hd}_{k}\left(N_{1}\right)=h\left(N_{1}\right)$ (and that $\mathrm{hd}_{k}\left(G_{1} / N_{1}\right)=h\left(G_{1} / N_{1}\right)$ but we do not need this). The final statement of our lemma now follows by combining with the equalities $\operatorname{hd}_{k}(N)=\operatorname{hd}_{k}\left(N_{1}\right)$ and $h(N)=h\left(N_{1}\right)$.

As remarked at the end of Section 1 this has the following consequence. 
Corollary 4.5. Let $G$ be an elementary amenable group with $\mathrm{hd}_{k}(G)=h(G)<\infty$. If $H$ is a subnormal subgroup of $G$, then $\operatorname{hd}_{k}(H)=h(H)<\infty$.

Of course, the corollary is also a consequence of Theorem A, but its role in the proof of Theorem A is significant. In the light of this, following some basic reductions the proof of Theorem A is mainly concerned with embedding a group as a subnormal subgroup of a locally $\mathrm{FP}_{\infty}$ group.

\section{Cohomological vanishing results, splittings, and Theorem B}

We shall need the following key vanishing result of Robinson in the special case when $S=\mathbb{Q}$ and $\operatorname{dim}_{\mathbb{Q}} M<\infty$.

Theorem 5.1 ([21, 10.3.1 and 10.3.2]). Let $S$ be a commutative ring, $G$ a nilpotent group, and $M$ an $S G$-module. If either

- $M$ is noetherian and $M_{G}=0$, or

- $M$ is artinian and $M^{G}=0$,

then $H^{n}(G, M)=H_{n}(G, M)=0$ for every $n$.

The proof of Theorem B. We are now in a position to prove the technical splitting theorem that is required for our solution to the homological dimension calculation.

Notational remark. In the following proof we write $A^{B}$ to indicate the set of $B$-fixed points in $A$ in case $B$ is a group acting on $A$. We use the right-handed convention for conjugation: $x^{y}=y^{-1} x y$ and note that $[x, y]=x^{-1} x^{y}$.

Proof of Theorem B. We proceed by induction on $h:=h(M)$. If $h=0$, then $M$ is trivial and we set $Q_{0}:=Q$ : note that (iv) implies $P$ is nilpotent in this case and the result is immediate. Suppose now that $h>0$. Choose $K$ to be a non-trivial divisible subgroup of $M$ of least possible Hirsch length subject to being normalized by $Q$. Note that $K$ must be abelian and therefore can be viewed as a $\mathbb{Q} Q$-module. As such $K$ is irreducible and the action of $Q$ descends to and action of $Q / K$. By induction, there is a subgroup $Q_{1}$ of $Q$ such that

- $L K \subseteq P \cap Q_{1}$,

- $\left(P \cap Q_{1}\right) / K$ is nilpotent, and

- $Q_{1} M=Q$.

At an important later step in this proof we shall want to restrict the action of $Q$ to $M$ and view $K$ as a $\mathbb{Q} M$-module. The choice of $K$ ensures that $K$ lies in the centre of $M$. We write $P_{1}:=P \cap Q_{1}$. As $M P_{1}=P \cap Q_{1} M=P \cap Q=P$ and $K^{M}=K$, we deduce

$$
K^{P}=K^{P_{1}} \text {. }
$$

The fact that $P$ is normal in $Q$ implies that $K^{P}$ is a $\mathbb{Q} Q$-submodule of $K$ thus the irreducibility of $K$ implies that either $K^{P}=K^{P_{1}}=0$ or $K^{P}=K^{P_{1}}=K$. In the later case, 
as $P_{1} / K$ is nilpotent, we deduce that also $P_{1}$ is and we only have to take $Q_{0}:=Q_{1}$. Then $Q_{0} \cap P=Q_{1} \cap P=P_{1}$ is nilpotent, $L \leq Q_{0}$ and $Q_{0} M=Q_{1} M=Q$. If we have the extra hypothesis on the finite generation of $Q / M$ and $L$, then in the inductive step we also deduce that $Q_{1} / K$ is finitely generated. Choose a set of lifts $X$ to $Q_{1}$ of a finite generating system of $Q_{1} / K$ and a finite generating system $Y$ of $L$ and let now $Q_{0}$ be the subgroup of $Q_{1}$ generated by $X \cup Y$. Then $Q_{0}$ is finitely generated and $L \leq Q_{0}$. Also, $Q_{0} \leq Q_{1}$ thus $Q_{0} \cap P \leq Q_{1} \cap P=P_{1}$ is nilpotent. Finally, by construction $Q_{0} K=Q_{1}$ thus

$$
Q_{0} M=Q_{0} K M=Q_{1} M=Q .
$$

So we may assume now that $K^{P}=K^{P_{1}}=0$. Then Theorem 5.1 shows that

$$
H^{*}\left(P_{1} / K, K\right)=0
$$

Using the spectral sequence $H^{*}\left(Q_{1} / P_{1}, H^{*}\left(P_{1} / K, K\right)\right) \Rightarrow H^{*}\left(Q_{1} / K, K\right)$ with $(\dagger)$, we find that $H^{*}\left(Q_{1} / K, K\right)$ also vanishes. In particular, $H^{2}\left(Q_{1} / K, K\right)=0$ and there is a splitting: there exists a group $Q_{0}$ of $Q_{1}$ such that $Q_{0} K=Q_{1}$ and $Q_{0} \cap K=\{1\}$. Note that $P_{1} \cap Q_{0}$ is a complement to $K$ in $P_{1}$ and is therefore nilpotent. Moreover, $Q_{0} M=Q_{1} K M=Q$. In the case when $L=1$ there is nothing else to prove.

So we assume now $L M=P$. At this point, we only need to establish the conclusion $L \subseteq Q_{0}$, and while this may not be true of the $Q_{0}$ we are currently entertaining, we show next that we can replace $Q_{0}$ with a conjugate subgroup to achieve our goal. We begin by observing that, since $M$ centralizes $K$, we have $K^{L}=K^{P}=1$. Because $L$ is nilpotent, this means $L \cap K=1$; that is, $L$ is a complement to $K$ in $P_{1}$. But all such complements must be conjugate because $H^{1}\left(P_{1} / K, K\right)=0$. Thus there is a $k \in K$ such that $\left(P_{1} \cap Q_{0}\right)^{k}=L$. Hence we can replace $Q_{0}$ with $Q_{0}^{k}$ to ensure that $L \subseteq Q_{0}$.

To finish the proof, note that if we have the extra hypothesis on finite generation, then $Q_{1} / K=Q_{0} K / K \cong Q_{0}$ is finitely generated.

\section{Integral automorphisms and Theorem $\mathrm{C}$}

Integral automorphisms play a role in Theorem $\mathrm{C}$ and also in the next section where we prove a special case of Theorem A. We therefore establish some general theory here.

Definition 6.1. Let $A$ be an abelian group. An endomorphism $\alpha$ of $A$ is algebraic (resp. integral) if there is a polynomial (resp. monic polynomial) $f \in \mathbb{Z}[x]$ such that $f(\alpha)=0$. Let $N$ be a nilpotent group. We say that an endomorphism of $N$ is integral if it induces an integral endomorphism on $N_{\mathrm{ab}}$.

The following two lemmas are elementary and the proofs are omitted.

Lemma 6.2. Let $\alpha$ be an endomorphism of an abelian group $A$ and $B$ a subgroup of $A$ such that $\alpha(B) \subseteq B$. Then $\alpha$ is integral if and only if it induces integral endomorphisms of both $B$ and $A / B$.

Lemma 6.3. Let $A$ be an abelian group and $\alpha \in \operatorname{End}(A)$. If $\alpha$ is algebraic, then there is an integer $m_{1} \geq 1$ such that $m \alpha$ is integral for every multiple $m$ of $m_{1}$. 
Lemma 6.4. Let $N$ be a nilpotent group and let $\alpha$ be an integral endomorphism of $N$. If $H$ is a subgroup of $N$ such that $\alpha(H) \subseteq H$, then the restriction of $\alpha$ to $H$ is integral.

Proof. This follows by the same reasoning used to establish [18, Lemma 3.24 (ii)].

Proof of Theorem C. The key results from Bieri and Stebel that we will invoke are [4, Theorem 4.6] and [5, Theorem A (iii)]. The notation and terminology we employ are also from those two articles. Let $Y$ be an arbitrary finite subset of $G$ and set $H=\langle X \cup Y\rangle$. We will show that $H$ is of type $\mathrm{FP}_{\infty}$. Put $Q=G / N$ and let $\varepsilon: G \rightarrow Q$ be the quotient map. Take $X_{0}$ to be a subset of $X$ that is mapped by $\varepsilon$ to a maximal linearly independent subset of $Q$. Let $Q_{0}$ be the subgroup of $Q$ generated by $\varepsilon\left(X_{0}\right)$. Note that $Q_{0}$ is then a free abelian group of finite index in $Q$ and $\varepsilon$ restricts to an epimorphism $H \rightarrow Q$ with kernel $H \cap N$. Since the elements of $X_{0}$ induce integral automorphisms on $N$, they also induce integral automorphisms on $H \cap N$ by Lemma 6.4. Let $A:=(H \cap N)_{\mathrm{ab}}$. According to [4, Theorem 4.6], the Bieri-Strebel invariant $\Sigma_{A}^{c}$ is contained in the open hemisphere $H_{q}=\{[v]: v(q)>0\}$ for $q$ equal to the product of the elements of $\varepsilon\left(X_{0}\right)$. Therefore by [5, Theorem A(iii)], $H$ is of type $\mathrm{FP}_{\infty}$.

\section{A special case of Theorem A}

Our goal in this section is to prove Theorem A in the special case when $G$ has the following structure: $G$ is a semidirect product $S \ltimes N$, where

- $N$ is a free nilpotent Mal'cev complete group of finite Hirsch length,

- $S$ is a subgroup of $\operatorname{Aut}(N)$ whose derived subgroup $\gamma_{2}(S)$ is contained in the group $U$ of 1-powering automorphisms of $N$,

- $S$ is finitely generated and nilpotent.

We need a preliminary result.

Proposition 7.1. Let $N$ be a Mal'cev complete nilpotent group of finite Hirsch length. Let $U$ be the group of 1-powering automorphisms of $N$. For $\alpha \in \operatorname{Aut}(N)$, there is some integer $m_{1} \geq 1$ such that for any multiple $m$ of $m_{1}$ and any m-powering automorphism $\theta$ of $N, \theta \alpha$ and the automorphism of $U$ induced by it are both integral.

Proof. Let $c$ be the nilpotency class of $N$. For each $2 \leq j \leq c$ let

$$
\beta_{j, \alpha}: \gamma_{j}(N) / \gamma_{j+1}(N) \rightarrow \gamma_{j}(N) / \gamma_{j+1}(N)
$$

be the automorphism induced by $\alpha$ and for $\left.B_{j}=\operatorname{Hom}\left(N_{\mathrm{ab}}, \gamma_{j}(N) / \gamma_{j+1}(N)\right)\right)$ let $\varphi_{j, \alpha}$ be the automorphism of $B_{j}$ induced by the diagonal action of $\alpha$, i.e.

$$
\varphi_{j, \alpha}: B_{j} \rightarrow B_{j}, \quad \xi \mapsto \beta_{j, \alpha} \xi \alpha^{-1}
$$

As all of $N_{\mathrm{ab}}$ and $B_{j}, 2 \leq j \leq c$ are $\mathbb{Q}$-vector spaces of finite dimension, all the automorphisms $\varphi_{j, \alpha}$ are algebraic and so is the automorphism of $N_{\mathrm{ab}}$ induced by $\alpha$ which we also denote $\alpha$ to avoid complications. By Lemma 6.3 taking the least common multiple of the integers involved, we may find an integer $m_{1}$ such that for any multiple $m$ of $m_{1}$, all the automorphisms $m \alpha, m \varphi_{j, \alpha}$ for $2 \leq j \leq c$ are integral. Now, let $\theta$ be an $m$-powering automorphism 
of $N$ and $\alpha_{1}=\theta \alpha$. The map of the $j$-th fold tensor product of $N_{\mathrm{ab}}$ by itself induced by $\theta$ is just multiplication by $m^{j}$ and as this tensor product maps onto $\gamma_{j}(N) / \gamma_{j+1}(N)$ the same holds true for $\gamma_{j}(N) / \gamma_{j+1}(N)$. This implies that $\beta_{j, \alpha_{1}}=m^{j} \beta_{j, \alpha}$. Hence $\varphi_{j, \alpha_{1}}=m^{j-1} \varphi_{j, \alpha}$, which means that $\varphi_{j, \alpha_{1}}$ is integral for $2 \leq j \leq c$.

Then Lemma 6.2 and Proposition 3.5 imply that the automorphism of $U_{j-1} / U_{j} \leq B_{j}$ induced by $\alpha_{1}=\theta \alpha$ is also integral. Let $\bar{U}_{j}$ be the image of $U_{j}$ in $U_{\mathrm{ab}}$. Then using the fact that $U_{c}=1$ because $N$ is nilpotent of class $c$ (see Proposition 3.5) we have a series

$$
U_{\mathrm{ab}}=\bar{U}_{1} \geq \bar{U}_{2} \geq \cdots \geq \bar{U}_{c}=0
$$

such that the automorphism of each factor induced by conjugating by $\alpha_{1}$ is integral. Hence the second part of the conclusion of the proposition follows from Lemma 6.2.

Proof that Theorem A holds in this special case. The group $S$ is finitely generated. Let $\left\{\alpha_{1}, \ldots, \alpha_{r}\right\}$ be a set of generators. Taking the least common multiple of the integers of Proposition 7.1 for each of the generators $\alpha_{i}$ we get an integer $m \geq 1$ such that for each $m$-powering automorphism $\varphi$ of $N, \varphi \alpha_{i}$ and the associated automorphism of $U$ are both integral, $1 \leq i \leq r$. Let $\varphi$ be an $m$-powering automorphism of $N$ and consider the group $W:=\langle U \cup\{\varphi\}\rangle$. Set $Q:=S W$ and $P:=S U$. Observe that $U$ is normal in $W$ and $W / U$ is cyclic and central in $Q / U$. Therefore the group $P$ is normal in $Q$. We now apply Theorem B with these groups $Q$ and $P$ and with $M:=U$, and $L:=S$. The output is a finitely generated subgroup, which we denote by $Q_{1}$, of $Q$ such that $Q=Q_{1} U, S \leq Q_{1}$, and $Q_{1} \cap P$ is nilpotent. Now all subgroups of a nilpotent group are subnormal and so $S$ is subnormal in $Q_{1} \cap P=Q_{1} \cap S U$. Therefore $S$ is subnormal in $Q_{1}$ and it follows that $S \ltimes N$ is subnormal in $Q_{1} \ltimes N$. By Corollary 4.5 , we have reduced to showing that

$$
\operatorname{hd}_{k}\left(Q_{1} \ltimes N\right)=h\left(Q_{1} \ltimes N\right) .
$$

The result follows from Proposition 4.3 because, as we shall now see, the group $Q_{1} \ltimes N$ is locally of type $\mathrm{FP}_{\infty}$.

The group $Q_{1}$ contains an $m$-powering automorphism $\theta$ (equal to $\varphi$ module $U$ ). Moreover, $Q_{1}$ is generated by the set $\left\{\alpha_{1}, \ldots, \alpha_{r}, \theta\right\} \cup Y$, where $Y$ is a finite subset of $U$. So it is also generated by the set $X:=\left\{\theta \alpha_{1}, \ldots, \theta \alpha_{r}, \theta\right\} \cup Y$. By Proposition 7.1 both $\theta \alpha_{i}$ and the automorphism of $U$ that it induces are integral for each $i$. Notice that the group $M:=\left(Q_{1} \cap U\right) \ltimes N$ is nilpotent and $Q_{1} \ltimes N$ is generated by $M \cup X$. Also by Lemma 6.4 the automorphisms of $Q_{1} \cap U$ induced by conjugating by the elements of $X$ are integral, which implies that the same is true of the automorphisms induced on $M$. Furthermore, since $S^{\prime} \leq U$, we have $Q^{\prime} \leq U$ which means that $Q_{1} /\left(Q_{1} \cap U\right)$ is abelian. Therefore we may apply Theorem C to this setup to deduce that $Q_{1} \ltimes N$ is locally of type $\mathrm{FP}_{\infty}$.

\section{Proof of Theorem A}

Let $G$ be a group with $\operatorname{hd}_{k}(G)<\infty$. By Lemma 4.1, $G$ has finite Hirsch length. Therefore by Lemma $2.1 G$ has a locally finite normal subgroup $T$ such that $G / T$ is torsion free nilpotent-by-free abelian of finite rank-by finite. We know that (see Proposition 4.2)

- $\operatorname{hd}_{k}(G)=\operatorname{hd}_{k}(G / T)$ for any locally finite normal subgroup $T$ of $G$, and

- $\operatorname{hd}_{k}(G)=\operatorname{hd}_{k}(H)$ for any subgroup $H$ of finite index in $G$. 
Obviously, the same happens for the Hirsch length and therefore we can replace $G$ by a section which has a torsion-free nilpotent normal subgroup $E$ such that $A:=G / E$ is free abelian of finite rank. We can embed $G$ into a larger group $\widehat{G}$ fitting into the commutative diagram

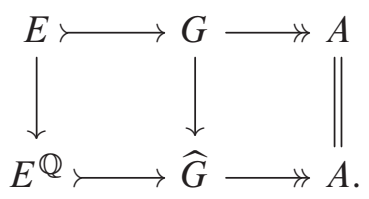

For the details of this construction see [15, Proposition 1.1]. We then have hd ${ }_{K}(G)=\operatorname{hd}_{K}(\widehat{G})$ and $h(G)=h(\widehat{G})$ thus we can replace $G$ by $\widehat{G}$ and assume that $E$ is already Mal'cev complete.

Using Theorem B with $Q:=P:=G, M:=E$ and $L:=1$, we may find a finitely generated nilpotent (thus polycyclic) subgroup $H$ of $G$ so that $G=H E$. Consider now the semidirect product $H \ltimes E$. There is a surjective map $H \ltimes E \rightarrow G=H E$ sending $(h, e)$ to $h e$ so an application of Lemma 4.4 implies that we can reduce the problem to the group $H \ltimes E$. Now, let $C_{H}(E)$ be the kernel in $H$ of the conjugacy action on $E$. This subgroup is normal in $H \ltimes E$ and the quotient map is $H \ltimes E \rightarrow H / C_{H}(E) \ltimes E$. Put $X=H / C_{H}(E)$. As the group $H$ is polycyclic, so is $C_{H}(E)$ thus we have that

$$
\operatorname{hd}_{K}(H \ltimes E)=\operatorname{hd}_{K}(X \ltimes E)+\operatorname{hd}_{K}\left(C_{H}(E)\right)
$$

which together with the fact that

$$
\operatorname{hd}_{K}\left(C_{H}(E)\right)=h\left(C_{H}(E)\right)
$$

because it is polycyclic implies that we can further reduce the problem to the group $X \ltimes E$. As $X$ acts faithfully on $E$, we may see it as a subgroup of the group $\operatorname{Aut}(E)$. From this point of view, $[X, X]$ consists exclusively of 1-powering automorphisms of $E$.

Using Proposition 3.6, we choose a free Mal'cev complete group $\widehat{E}$ with the same class so that there is a surjective homomorphism $\pi: \widehat{E} \rightarrow E$ that induces an isomorphism

$$
\widehat{E} / \gamma_{2}(\widehat{E}) \cong E / \gamma_{2}(E) \text {. }
$$

There is an induced epimorphism

$$
\bar{\pi}: \operatorname{Aut}(\widehat{E} ; \operatorname{Ker} \pi) \rightarrow \operatorname{Aut}(E)
$$

whose kernel $Z$ is contained in the subgroup $U(\operatorname{Aut}(\widehat{E}))$ of 1-powering automorphisms. Note that $Z$ is nilpotent and by Lemma 3.7 Mal'cev complete.

Let $T$ denote the preimage of $X$ under the map $\bar{\pi}$. From Proposition 3.6(ii) we see that $[T, T]$ consists entirely of 1-powering automorphisms of $\widehat{E}$. At this point we may apply again Theorem B with $T$ playing the role of both $P$ and $Q$, with $M:=Z$ and with $L:=\{1\}$ and we deduce that there is some $S \leq T$ finitely generated and nilpotent such that $S Z=T$. We can now consider the semidirect product $S \ltimes \widehat{E}$ which collapses naturally via $\pi$ and $\bar{\pi}$ onto $X \ltimes E$. By Lemma 4.4 it suffices to prove that

$$
\operatorname{hd}_{k}(S \ltimes \widehat{E})=h(S \ltimes \widehat{E}) .
$$

We have reduced to the special case that was considered in preceding section and Theorem A follows. 


\section{References}

[1] R. Baer, Polyminimaxgruppen, Math. Ann. 175 (1968), 1-43.

[2] G. Baumslag and R. Bieri, Constructable solvable groups, Math. Z. 151 (1976), no. 3, 249-257.

[3] R. Bieri, Homological dimension of discrete groups, 2nd ed., Queen Mary College Math. Notes, Queen Mary College, London 1981.

[4] R. Bieri and R. Strebel, A geometric invariant for modules over an abelian group, J. reine angew. Math. 322 (1981), 170-189.

[5] R. Bieri and R. Strebel, A geometric invariant for nilpotent-by-abelian-by-finite groups, J. Pure Appl. Algebra 25 (1982), no. 1, 1-20.

[6] M.R. Bridson and P. H. Kropholler, Dimension of elementary amenable groups, J. reine angew. Math. 699 (2015), 217-243.

[7] K. S. Brown and R. Geoghegan, Cohomology with free coefficients of the fundamental group of a graph of groups, Comment. Math. Helv. 60 (1985), no. 1, 31-45.

[8] A.S. Detinko, D. L. Flannery and E.A. O'Brien, Algorithms for linear groups of finite rank, J. Algebra 393 (2013), 187-196.

[9] G. L. Fel'dman, The homological dimension of group algebras of solvable groups, Izv. Akad. Nauk SSSR Ser. Mat. 35 (1971), 1225-1236.

[10] D. Gildenhuys and R. Strebel, On the cohomology of soluble groups. II, J. Pure Appl. Algebra 26 (1982), no. 3, 293-323.

[11] J. A. Hillman, Elementary amenable groups and 4-manifolds with Euler characteristic 0, J. Aust. Math. Soc. Ser. A 50 (1991), no. 1, 160-170.

[12] J. A. Hillman, Four-manifolds, geometries and knots, Geom. Topol. Monogr. 5, Geometry \& Topology Publications, Coventry 2002.

[13] J.A. Hillman and P.A. Linnell, Elementary amenable groups of finite Hirsch length are locally-finite by virtually-solvable, J. Aust. Math. Soc. Ser. A 52 (1992), no. 2, 237-241.

[14] P. Hilton, Localization and cohomology of nilpotent groups, Math. Z. 132 (1973), 263-286.

[15] P. Hilton, Relative nilpotent groups, in: Categorical aspects of topology and analysis Ottawa 1980, Lecture Notes in Math. 915, Springer, Berlin (1982), 134-147.

[16] P. H. Kropholler, Cohomological dimension of soluble groups, J. Pure Appl. Algebra 43 (1986), no. 3, 281-287.

[17] P. H. Kropholler, On groups of type (FP) $\infty$, J. Pure Appl. Algebra 90 (1993), no. 1, 55-67.

[18] P. H. Kropholler and K. Lorensen, Virtually torsion-free covers of minimax groups, Ann. Sci. Éc. Norm. Supér. (4), to appear.

[19] P. H. Kropholler, C. Martinez-Pérez and B. E. A. Nucinkis, Cohomological finiteness conditions for elementary amenable groups, J. reine angew. Math. 637 (2009), 49-62.

[20] P. H. Kropholler and G. Mislin, Groups acting on finite-dimensional spaces with finite stabilizers, Comment. Math. Helv. 73 (1998), no. 1, 122-136.

[21] J. C. Lennox and D. J.S. Robinson, The theory of infinite soluble groups, Oxford Mathematical Monographs, The Clarendon Press, Oxford 2004.

[22] U. Stammbach, On the weak homological dimension of the group algebra of solvable groups, J. Lond. Math. Soc. (2) 2 (1970), 567-570.

[23] B. A. F. Wehrfritz, On elementary amenable groups of finite Hirsch number, J. Aust. Math. Soc. Ser. A 58 (1995), no. 2, 219-221.

Peter H. Kropholler, Mathematical Sciences, University of Southampton, Southampton, United Kingdom

https://orcid.org/0000-0001-5460-1512

e-mail: p.h.kropholler@soton.ac.uk

Conchita Martínez-Pérez, University of Zaragoza, Zaragoza, Spain

https://orcid.org/0000-0001-9147-1745

e-mail: conmar@unizar.es

Eingegangen 25. Mai 2018, in revidierter Fassung 7. Januar 2019 